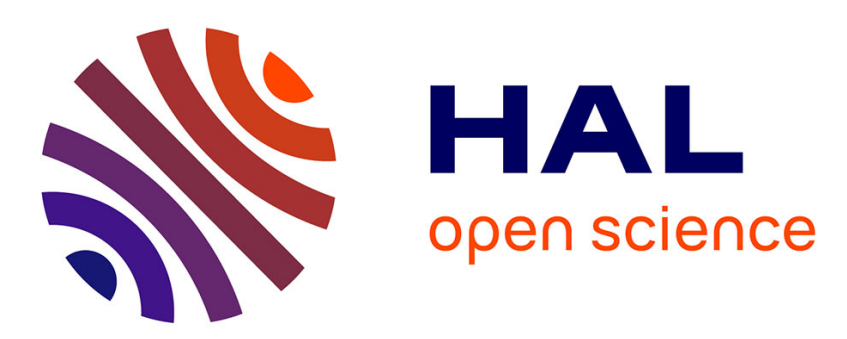

\title{
Observer synthesis for uncertain nonlinear systems. Application to waste-water treatment plants
}

Anca Maria Nagy-Kiss, Benoît Marx, Gilles Mourot, Georges Schutz, José Ragot

\section{- To cite this version:}

Anca Maria Nagy-Kiss, Benoît Marx, Gilles Mourot, Georges Schutz, José Ragot. Observer synthesis for uncertain nonlinear systems. Application to waste-water treatment plants. 7th IFAC Symposium on Robust Control Design, ROCOND'12, Jun 2012, Aalborg, Denmark. pp.485-490, 10.3182/20120620-3-DK-2025.00104 . hal-00679563

\section{HAL Id: hal-00679563 https://hal.science/hal-00679563}

Submitted on 26 Nov 2021

HAL is a multi-disciplinary open access archive for the deposit and dissemination of scientific research documents, whether they are published or not. The documents may come from teaching and research institutions in France or abroad, or from public or private research centers.
L'archive ouverte pluridisciplinaire HAL, est destinée au dépôt et à la diffusion de documents scientifiques de niveau recherche, publiés ou non, émanant des établissements d'enseignement et de recherche français ou étrangers, des laboratoires publics ou privés. 


\title{
Observer synthesis for uncertain nonlinear systems. Application to waste-water treatment plants
}

\author{
Anca Maria Nagy-Kiss* Benoît Marx ${ }^{* *}$ Gilles Mourot ${ }^{* *}$ \\ Georges Schutz* José Ragot ${ }^{* *}$ \\ * Unit of Modelling and Simulation, Public Research Center Henri Tudor, 29, \\ avenue John F. Kennedy L-1855 Luxembourg-Kirchberg, Luxembourg \\ (e-mail: anca-maria.nagy,georges.schutz@tudor.lu). \\ ** Centre de Rescherche en Automatique de Nancy, INPL, 2, avenue de la \\ forêt de Haye, 54500 Vandoeuvre-lès-Nancy, France (e-mail: benoit.marx, \\ gilles.mourot,jose.ragot@ensem.inpl-nancy.fr)
}

\begin{abstract}
The paper proposes an observer synthesis for uncertain nonlinear systems, described by multimodels with unmeasurable premise variables, affected by unknown inputs. A proportional multi-integral observer is considered in order to estimate the system states and the unknown inputs and minimize the influence of the model uncertainties. The stability analysis and the observer synthesis are expressed through linear matrix inequalities based on the Lyapunov method. The performances of the proposed observer synthesis method are highlighted through the application to a waste-water treatment plant model, which is an uncertain nonlinear system affected by unknown inputs.
\end{abstract}

\section{INTRODUCTION}

Environmental or technological systems have complex behaviors. Thus, their representation in a large operating domain involves nonlinear relations between the process variables, the system parameters, the control inputs and the external perturbations. On the other hand, in the field fields of observer design for fault diagnosis or fault tolerant control, the extension of linear methods to nonlinear systems is generally a difficult problem. Thus, it is a need to build systems that can operate over a wide range of operating conditions, such as models based on a decomposition of the system model into a number of simpler linear models. Multi-model (MM) has proven to be a powerful tool in the representation of nonlinear systems on a compact set of the state space, as mentioned in chap. 14 of Tanaka and Wang [2001] and also in the analysis and synthesis of a nonlinear control system Murray-Smith and Johansen [1997], Angelis [2001].

Several techniques for obtaining MM were developed Johansen et al. [2000], Tanaka and Wang [2001], Nagy et al. [2010]. The sector nonlinearity approach Tanaka and Wang [2001] allows to exactly rewrite a nonlinear system into a MM, but the choice of the decision variables has not been systematically realized. A systematic multi-modeling rewriting with a motivated choice of these variables is presented in Nagy et al. [2010] which also allows to avoid the model linearization and its drawbacks. This last method will be used here for obtaining the MM.

This paper mainly focuses on the use of multi-models for observer synthesis Bergsten et al. [2002], Marx et al. [2007]. The observer design represents, in the last decades, an active research field owing to its particular importance in observerbased control, fault diagnosis and fault tolerant control Tanaka and Wang [2001], Chen and Saif [2007], Koenig and Mammar [2002], Ichalal et al. [2010].

Most of the existing works, dedicated to observer design for $\mathrm{MM}$, are established for MM with measurable decision vari- ables (inputs/outputs), that represents a simplified situation Tanaka and Wang [2001], Marx et al. [2007]. The MM under study in this paper involves unmeasurable decision variables depending on the state variables -frequently met in practical situations- that are not always accessible.

A proportional multi-integral (PMI) observer approach NagyKiss et al. [2011] for uncertain nonlinear systems with unknown inputs presented under a MM form is proposed in this paper. The state and unknown input estimation given by this observer is made simultaneously and the influence of the model uncertainties is minimized through a $\mathscr{L}_{2}$ gain. The convergence conditions of the state and unknown input estimation errors are expressed through LMIs (Linear Matrix Inequalities) Boyd et al. [1994], Tanaka and Wang [2001] by using the Lyapunov method and the $\mathscr{L}_{2}$ approach. PMI observers were previously proposed by Jiang et al. [2000] for linear systems, by Koenig [2005], Gao and Ho [2004] in order to estimate a large class of polynomial signals for LTI descriptor systems and by Ichalal et al. [2009] to estimate state and unknown inputs of nonlinear systems expressed under Takagi-Sugeno form but without considering uncertainties affecting the system. Therefore, this motivates us to derive a novel PMI observer technique that handles the uncertainties influence on the estimation error.

The practical contribution of this paper is to apply the proposed modeling and observer method to the realistic model of a waste-water treatment process (WWTP) modeled by an ASM1 nonlinear model with ten states, that is equivalently rewritten as a MM. The measures used for simulation process are those of the European program benchmark Cost 624 Coop [2002]. The choice of the known/unknown inputs, the measures and the real conditions is made by taking into account the properties of the Bleesbruck treatment station from Luxembourg. The numerical simulation results of the considered application show good state and unknown inputs estimation performances.

Section 2 presents the multi-modeling approach and give the problem statement. Section 3 gives the proposed observer syn- 
thesis. Some results and performances of the proposed observer are illustrated in section 4 through a complex model of WWTP. Conclusions and future works are given in the end of this proposal.

\section{MULTI-MODELLING APPROACH}

Generally, a nonlinear system can be described by:

$$
\begin{aligned}
& \dot{x}(t)=f(x(t), u(t), d(t)) \\
& y(t)=g(x(t), u(t), d(t))
\end{aligned}
$$

The MM approach allows to represent any nonlinear dynamic system with uncertainties and affected by unknown inputs in a compact set of the state space with a convex combination of linear sub-models Nagy et al. [2010]:

$$
\begin{aligned}
\dot{x}(t)= & \sum_{i=1}^{r} \mu_{i}(x, u)\left[\left(A_{i}+\Delta A_{i}(t)\right) x(t)+E_{i} d(t)\right. \\
& \left.+\left(B_{i}+\Delta B_{i}(t)\right) u(t)\right] \\
y(t)= & C x(t)+G d(t) \\
\sum_{i=1}^{r} \mu_{i}(x, u)= & 1, \mu_{i}(x, u) \geq 0, \forall(x, u) \in \mathbb{R}^{n} \times \mathbb{R}^{n_{u}}
\end{aligned}
$$

where $x(t) \in \mathbb{R}^{n}$ is the system state, $u(t) \in \mathbb{R}^{n_{u}}$ is the known input, $d(t) \in \mathbb{R}^{n_{d}}$ is the unknown input, $y(t) \in \mathbb{R}^{n_{y}}$ is the measured output and the matrices of appropriate dimensions are known and constant excepted $\Delta A_{i}(t)$ and $\Delta B_{i}(t)$ that satisfy the following equations

$$
\begin{array}{llll}
\Delta A_{i}(t) & =M_{i}^{a} F_{a}(t) N_{i}^{a}, & \text { with } & F_{a}^{T}(t) F_{a}(t) \leq I \\
\Delta B_{i}(t)=M_{i}^{b} F_{b}(t) N_{i}^{b}, & \text { with } & F_{b}^{T}(t) F_{b}(t) \leq I
\end{array}
$$

where both $F_{a}(t) \in \mathbb{R}^{f_{1} \times f_{1}}$ and $F_{b}(t) \in \mathbb{R}^{f_{2} \times f_{2}}$ are unknown and time varying and $M_{i}^{a}, M_{i}^{b}, N_{i}^{a}$ and $N_{i}^{b}$ are known matrices of appropriate dimensions. One can note that the activating functions $\mu_{i}$ depend on the system state that is not available to the measurement.

In the sequel, the following assumption is made:

Hypothesis 1. The unknown input $d \in C^{q}$ is assumed to be a bounded time varying signal with null $q^{\text {th }}$ derivative:

$$
d^{(q)}(t)=0
$$

In proportional integral (PI) observer design, the unknown input must be constant $(\dot{d}(t)=0)$ in order to prove the estimation error convergence (Koenig and Mammar [2002]). This first hypothesis still gives good result if the unknown inputs vary slowly. Although, for fast variations of the unknown input no good estimation are obtained. Then, PMI observer is more adequate for this problem, because the observer estimates the $(q-1)^{t h}$ derivatives of the unknown input and gives a good precision for the estimation of the unknown inputs as in Ichalal et al. [2009]. For instance, one will see, in section 4, that good estimation results are obtained with this last hypothesis 1 .

\section{OBSERVER DESIGN FOR UNCERTAIN NONLINEAR SYSTEMS}

In order to estimate both the system state and the unknown input, the following PMI Observer is proposed:

$$
\begin{aligned}
\dot{\hat{x}}(t)= & \sum_{i=1}^{r} \mu_{i}(\hat{x}(t), u(t))\left(A_{i} \hat{x}(t)+B_{i} u(t)+E_{i} \hat{d}(t)\right. \\
& \left.+L_{P i}(y(t)-\hat{y}(t))\right) \\
\dot{\hat{d}}_{j}(t)= & \sum_{i=1}^{r} \mu_{i}(\hat{x}(t), u(t)) L_{I i}^{j}(y(t)-\hat{y}(t))+\hat{d}_{j+1} \\
\hat{y}(t)= & C \hat{x}(t)+G \hat{d}(t)
\end{aligned}
$$

for $j=1, \ldots, q-1$ par $j=0, \ldots, q-1$, where $\hat{d}_{i}, i=0, \ldots, q-1$ are the estimates of $d(t)$ and its $(q-1)$ first derivatives. The state and unknown inputs estimation errors are:

$$
e=x-\hat{x}, e_{0}=\dot{d}-\dot{\hat{d}}_{0}, \ldots, e_{q-1}=\dot{d}_{q-1}-\dot{\hat{d}}_{q-1}
$$

The observer design reduces to find the gains $L_{I i}=\left[\begin{array}{llll}L_{I i}^{0 T} & L_{I i}^{1 T} & \cdots & L_{I i}^{q-1 T}\end{array}\right]^{T}$ and $L_{P i}$ s.t. the state and unknown input estimation error obey to a stable system.

Notation 3.1. The symbol $*$ in a block matrix denotes the blocks induced by symmetry. For any square matrix $M, \mathbb{S}(M)$ is defined by $\mathbb{S}(M)=M+M^{T}$.

Theorem 2. The observer (5) estimating the state and unknown input of the system (2) and minimizing the $\mathscr{L}_{2}$-gain $\gamma$ of the known and unknown inputs on the state and unknown input estimation error is obtained by finding symmetric positive definite matrices $P_{1} \in \mathbb{R}^{\left(n+q \cdot n_{d}\right) \times\left(n+q \cdot n_{d}\right)}$ and $P_{2} \in \mathbb{R}^{n \times n}$, matrices $\bar{P}_{j} \in$ $\mathbb{R}^{\left(n+q \cdot n_{d}\right) \times n_{y}}$ and positive scalars $\varepsilon_{1 i}$ and $\varepsilon_{2 i}$ for all $i=1, \cdots, r$ that minimize the scalar $\bar{\gamma}$ under the following LMI constraints

$$
\mathscr{M}_{i j}<0, i, j=1, \ldots, r
$$

where $\mathscr{M}_{i j}$ is defined by

$$
\mathscr{M}_{i j}=\left[\begin{array}{cccccc}
\Theta_{i j}^{11} & \Theta_{i j}^{12} & \Theta_{i j}^{13} & \Theta_{i j}^{14} & P_{1} \bar{M}_{i}^{a} & P_{1} \bar{M}_{i}^{b} \\
* & \Theta_{i j}^{22} & P_{2} B_{i} & P_{2} E_{i} & P_{2} M_{i}^{a} & P_{2} M_{i}^{b} \\
* & * & \Theta_{i j}^{33} & 0 & 0 & 0 \\
* & * & * & -\bar{\gamma} I_{n_{d}} & 0 & 0 \\
* & * & * & * & -\varepsilon_{1 i} I_{f_{1}} & 0 \\
* & * & * & * & * & -\varepsilon_{2 i} I_{f_{2}}
\end{array}\right]
$$

$$
\begin{array}{ll}
\Theta_{i j}^{11}=I_{n+q \cdot n_{d}}+\mathbb{S}\left(P_{1} \bar{A}_{j}-\bar{P}_{j} \bar{C}\right) & \Theta_{i j}^{12}=P_{1}\left(\tilde{A}_{i}-\tilde{A}_{j}\right) \\
\Theta_{i j}^{13}=P_{1}\left(\tilde{B}_{i}-\tilde{B}_{j}\right) & \Theta_{i j}^{14}=P_{1}\left(\tilde{E}_{i}-\tilde{E}_{j}\right) \\
\Theta_{i j}^{22}=\varepsilon_{1 i} N_{i}^{a T} N_{i}^{a}+\mathbb{S}\left(P_{2} A_{i}\right) & \Theta_{i j}^{33}=\varepsilon_{2 i} N_{i}^{b T} N_{i}^{b}-\bar{\gamma} I_{n_{u}}
\end{array}
$$

with

$$
\begin{gathered}
\bar{C}=\left[\begin{array}{c}
C^{T} \\
G^{T} \\
0 \\
\vdots \\
0
\end{array}\right], \bar{A}_{i}=\left[\begin{array}{cccccc}
A_{i} & E_{i} & 0 & 0 & \cdots & 0 \\
0 & 0 & I_{n_{d}} & 0 & \cdots & 0 \\
\vdots & \vdots & & \ddots & & \vdots \\
0 & 0 & & 0 & \cdots & I_{n_{d}} \\
0 & 0 & \cdots & 0 & \cdots & 0
\end{array}\right], \\
\tilde{A}_{i}=\left[\begin{array}{c}
A_{i} \\
0 \\
\vdots \\
0
\end{array}\right], \tilde{E}_{i}=\left[\begin{array}{c}
E_{i} \\
0 \\
\vdots \\
0
\end{array}\right], \bar{M}_{i}^{a}=\left[\begin{array}{c}
M_{i}^{a}(t) \\
0 \\
\vdots \\
0
\end{array}\right], \bar{M}_{i}^{b}=\left[\begin{array}{c}
M_{i}^{b}(t) \\
0 \\
\vdots \\
0
\end{array}\right]
\end{gathered}
$$

The $\mathscr{L}_{2}$-gain $\gamma$ is given by $\gamma=\sqrt{\gamma}$ and the observer gains are obtained by

$$
L_{j}=\left[\begin{array}{l}
L_{P j} \\
L_{I j}
\end{array}\right]=P_{1}^{-1} \bar{P}_{j}
$$

Proof. Let us define an augmented state and its estimate by

$$
\begin{aligned}
& x_{a}(t)=\left[\begin{array}{lllll}
x(t)^{T} & d(t)^{T} & d_{1}(t)^{T} & \cdots & d_{q-1}(t)^{T}
\end{array}\right]^{T} \\
& \hat{x}_{a}(t)=\left[\begin{array}{lllll}
\hat{x}(t)^{T} & \hat{d}(t)^{T} & \hat{d}_{1}(t)^{T} & \cdots & \hat{d}_{q-1}(t)^{T}
\end{array}\right]^{T}
\end{aligned}
$$


The augmented state estimation error is defined by $e_{a}(t)=$ $x_{a}(t)-\hat{x}_{a}(t)$. Using (2a) and (4), the system and observer equations can be respectively written as

$$
\begin{aligned}
\dot{x}_{a}(t)= & \sum_{i=1}^{r} \mu_{i}\left(x_{a}(t), u(t)\right)\left[\left(\bar{A}_{i}+\overline{\Delta A}_{i}(t)\right) x_{a}(t)\right. \\
& \left.+\left(\bar{B}_{i}+\overline{\Delta B}_{i}(t)\right) u(t)\right] \\
y(t)= & \bar{C} x_{a}(t)
\end{aligned}
$$

with :

$$
\begin{gathered}
\bar{B}_{i}=\left[\begin{array}{c}
B_{i} \\
0 \\
\vdots \\
0
\end{array}\right], \quad \bar{N}_{i}^{b T}=\left[\begin{array}{c}
N_{i}^{b T} \\
0 \\
\vdots \\
0
\end{array}\right], \quad \bar{N}_{i}^{a T}=\left[\begin{array}{c}
N_{i}^{a T} \\
0 \\
\vdots \\
0
\end{array}\right], \\
\overline{\Delta B}_{i}(t)=\bar{M}_{i}^{b} F^{b}(t) N_{i}^{b}, \quad \overline{\Delta A}_{i}(t)=\bar{M}_{i}^{a} F^{a}(t) \bar{N}_{i}^{a}
\end{gathered}
$$

and

$$
\begin{aligned}
\dot{\hat{x}}_{a}(t)= & \sum_{j=1}^{r} \mu_{j}\left(\hat{x}_{a}(t), u(t)\right)\left[\bar{A}_{j} \hat{x}_{a}(t)+\bar{B}_{j} u(t)\right. \\
& \left.+L_{j}(y(t)-\hat{y}(t))\right] \\
\hat{y}(t)= & \bar{C} \hat{x}_{a}(t)
\end{aligned}
$$

One should note that in (12a) the activating functions depend on $x_{a}(t)$, whereas they depend on $\hat{x}_{a}(t)$ in (14a) and then the comparison of the state $x_{a}(12 \mathrm{a})$ and its reconstruction (14a) seems to be difficult. In order to cope with the difficulty of expressing the augmented state estimation error in a tractable way, (12a) is re-written, based on the property (2c). Consequently, the augmented state estimation error obeys to the following nonlinear system

$$
\begin{aligned}
& {\left[\begin{array}{c}
\dot{e}_{a}(t) \\
\dot{x}(t)
\end{array}\right]=} \sum_{i=1}^{r} \sum_{j=1}^{r} \mu_{i}\left(x_{a}(t), u(t)\right) \mu_{j}\left(\hat{x}_{a}(t), u(t)\right) \\
& \cdot\left\{\mathscr{A}_{i j}(t)\left[\begin{array}{c}
e_{a}(t) \\
x(t)
\end{array}\right]+\mathscr{B}_{i j}(t)\left[\begin{array}{c}
u(t) \\
d(t)
\end{array}\right]\right\} \\
& e_{a}(t)=\left[\begin{array}{ll}
I_{n+n_{d}} & 0
\end{array}\right]\left[\begin{array}{c}
e_{a}(t) \\
x(t)
\end{array}\right]
\end{aligned}
$$

where

$$
\begin{aligned}
& \mathscr{A}_{i j}(t)=\left[\begin{array}{cc}
\bar{A}_{j}-L_{j} \bar{C} & \tilde{A}_{i}-\tilde{A}_{j}+\widetilde{\Delta A}_{i}(t) \\
0 & A_{i}+\Delta A_{i}(t)
\end{array}\right] \\
& \mathscr{B}_{i j}(t)=\left[\begin{array}{cc}
\overline{B_{i}}-\overline{B_{j}}+\overline{\Delta B}_{i}(t) & \tilde{E}_{i}-\tilde{E}_{j} \\
B_{i}+\Delta B_{i}(t) & E_{i}
\end{array}\right] \\
& \widetilde{\Delta A}_{i}(t)=\left[\begin{array}{llll}
\Delta A_{i}(t)^{T} & 0 & \cdots & 0
\end{array}\right]^{T}
\end{aligned}
$$

The candidate Lyapunov function for $(15)$ is

$$
V\left(x_{a}(t), x(t)\right)=\left[\begin{array}{c}
e_{a}(t) \\
x(t)
\end{array}\right]^{T}\left[\begin{array}{cc}
P_{1} & 0 \\
0 & P_{2}
\end{array}\right]\left[\begin{array}{c}
e_{a}(t) \\
x(t)
\end{array}\right]
$$

where $P_{1}$ and $P_{2}$ are symmetric positive definite matrices. The objective is to find the gains $L_{j}$ of the observer that minimize the $\mathscr{L}_{2}$-gain from the known and unknown inputs $u(t)$ and $d(t)$ to the state and unknown input estimation error $e_{a}(t)$. It is well known Boyd et al. [1994] that the $\mathscr{L}_{2}$-gain from $\left[\begin{array}{l}u(t) \\ d(t)\end{array}\right]$ to $e_{a}(t)$ is bounded by $\gamma$ if

$$
\dot{V}\left(e_{a}(t), x(t)\right)+e_{a}^{T}(t) e_{a}(t)-\gamma^{2}\left(u^{T}(t) u(t)+d^{T}(t) d(t)\right)<0
$$

With some Schur complements and defining $\bar{P}_{j}=P_{1} L_{j}$ and $\bar{\gamma}=\gamma^{2}$, the previous inequality becomes

$$
\sum_{i=1}^{r} \sum_{j=1}^{r} \mu_{i}\left(x_{a}(t), u(t)\right) \mu_{j}\left(\hat{x}_{a}(t), u(t)\right) \mathscr{M}_{i j}<0
$$

It follows that (20) is satisfied if the LMI (6) holds, which achieves the proof. For more details see Nagy-Kiss et al. [2011], where a PI observer was proposed for a reduced ASM1 model.

The proposed estimation method requires that the system must be quadratically robustly stable, as it can be seen in the block $(2,2)$ of the LMI $(6)$.

The performance analysis and the synthesis of observer for multi-model is based on the resolution of LMI by using the Lyapunov method. Because of the convex sum property of the weighting functions $(2 \mathrm{c})$, the LMI are only evaluated at the polytope vertices $\left(A_{i}, B_{i}\right)$ (or in the uncertain case adding the terms $\Delta A_{i}$ and $\Delta B_{i}$ ) and the weighting functions do not occur in the resolution of the LMIs. One should keep in mind that the LMI formulation of observer design for MM consists only in sufficient but not necessary conditions. That is the reason why it is essential to propose the appropriate MM structure. Detailed choice criteria for MM are discussed in Nagy et al. [2010]; it is to mention here the observability criteria, which means that MM structures with observable submodels are necessary. Thus, avoid submodel matrices $C_{i}$ with null rows is an observability requirement. It should be also mentioned that a MM with a low number of submodels is preferred, which leads to less computational requirements by reducing the number of LMI to be solved.

Remark 3.1. If the system is also affected by unknown input which estimates are not needed, the system (2) may be written as

$$
\begin{aligned}
\dot{x}(t)= & \sum_{i=1}^{r} \mu_{i}(x(t))\left[\left(A_{i}+\Delta A_{i}(t)\right) x(t)\right. \\
& \left.+\left(B_{i}+\Delta B_{i}(t)\right) u(t)+E_{i} d(t)+F_{i} w(t)\right] \\
y(t)= & C x(t)+G d(t)+H w(t)
\end{aligned}
$$

where $w(t) \in \mathbb{R}^{n_{w}}$ denotes the unknown inputs that are not to be estimated. In this case, the previous result can readily be adapted in order to estimate both $x(t)$ and $d(t)$ while minimizing the influence of $w(t)$ on the estimation errors. One should see that the matrices $\mathscr{M}_{i j}$ in (6) should be replaced by $\tilde{\mathscr{M}}_{i j}$ defined by

$$
\tilde{\mathscr{M}}_{i j}=\left[\begin{array}{cc}
\mathscr{M}_{i j} & \Psi_{i j} \\
* & -\bar{\gamma} I_{n_{w}}
\end{array}\right]
$$

with $\Psi_{i j}^{T}=\left[\left(\bar{P}_{j} \bar{H}-P_{1} \bar{F}_{i}\right)^{T} F_{i}^{T} P_{2} \quad 0 \quad 0\right], \bar{H}^{T}=\left[\begin{array}{ll}H^{T} & 0\end{array}\right]$ and $\bar{F}_{i}^{T}=\left[\begin{array}{ll}F_{i}^{T} & 0\end{array}\right]$.

\section{WASTE-WATER TREATMENT PLANT}

\subsection{Process description and ASM1 model}

The widely used activated sludge based waste-water treatment consists in mixing waste-waters with a rich mixture of bacteria in order to degrade the organic matter Olsson and Newell [1999].

In this work, a part of the COST Benchmark is considered. The Benchmark is based on the most common WWTP: a continuous flow activated sludge plant, performing nitrification and de-nitrification. A configuration with a single tank with a settler/clarifier was developed. The objective of this study is to use the data generated by this benchmark.

For observer/controller design, models of reduced complexity are generally used. Nevertheless, this paper considers a quite 
complete ASM1 model for WWTP involving the following components: soluble carbon $S_{S}$, particulate $X_{S}$, dissolved oxygen $S_{O}$, heterotrophic biomass $X_{B H}$, ammonia $S_{N H}$, nitrate $S_{N O}$, autotrophic biomass $X_{B A}$, soluble inert $S_{I}$, suspended inert $X_{I}$, soluble organic nitrogen $S_{N D}$ and suspended organic nitrogen $X_{N D}$. Only the following components are not considered in the ASM1 model: the inert component $X_{P}$ and the alkalinity $S_{a l k}$. As in practical situation, a single organic compound, denoted $X_{D C O}$, will be considered by adding the soluble part $S_{S}$ and the particulate part $X_{S}$ Smets et al. [2003]. The following state vector is taken:

$$
\begin{aligned}
x(t)= & {\left[X_{D C O}(t), S_{O}(t), S_{N H}(t), S_{N O}(t), X_{B H}(t) \cdots\right.} \\
& \left.\cdots X_{B A}(t), S_{I}(t), X_{I}(t), S_{N D}(t), X_{N D}(t)\right]^{T}
\end{aligned}
$$

The following assumptions are considered: the dissolved oxygen concentration input $\left(S_{O, \text { in }}\right)$ is null, $S_{N O, \text { in }} \cong 0$ and $X_{B A, \text { in }} \cong 0$, which is in conformity with the European Benchmark COST 624 Coop [2002].

In practice, the concentrations $X_{D C O, i n}, S_{N H, \text { in }}$ and $X_{B H, \text { in }}$ are not measured online. A daily mean value will be considered for $X_{D C O, \text { in }}$ and $X_{B H, i n}$. The concentration $S_{N H, i n}$ is considered as unknown input. The measurements of $\left(X_{D C O}, S_{O}, S_{N H}\right.$ and $S_{N O}$ ) are considered to be available online. Consequently, the output $y$, the known input $u$ and the unknown input $d$ vectors are:

$$
\begin{aligned}
y(t)= & {\left[X_{D C O}(t), S_{O}(t), S_{N H}(t), S_{N O}(t)\right]^{T} } \\
u(t)= & {\left[X_{D C O, \text { in }}(t), q_{a}(t), X_{B H, i n}, S_{I, \text { in }}(t), \cdots\right.} \\
& \left.\cdots X_{I, \text { in }}(t), S_{N D, \text { in }}(t), X_{N D, \text { in }}(t)\right]^{T} \\
d(t)= & S_{N H, \text { in }}(t)
\end{aligned}
$$

Let us consider the dynamic ASM1 model with the state vector (24):

$$
\begin{aligned}
& \dot{X}_{D C O}(t)=-\frac{1}{Y_{h}}\left[\varphi_{1}(t)+\varphi_{2}(t)\right]+\left(1-f_{p}\right)\left[\varphi_{4}(t)\right. \\
&\left.+\varphi_{5}(t)\right]+D_{1}(t) \\
& \dot{S}_{O}(t)=\frac{Y_{h}-1}{Y_{h}} \varphi_{1}(t)+\frac{Y_{a}-4.57}{Y_{a}} \varphi_{3}(t)+D_{2}(t) \\
& \dot{S}_{N H}(t)=-i_{x b}\left[\varphi_{1}(t)+\varphi_{2}(t)\right]-\left[i_{x b}+\frac{1}{Y_{a}}\right] \varphi_{3}(t) \\
& \quad+\left(i_{x b}-f_{p} i_{x p}\right)\left[\varphi_{4}(t)+\varphi_{5}(t)\right]+D_{3}(t) \\
& \dot{S}_{N O}(t)= \\
& \dot{X}_{B H}(t)=\varphi_{1}(t)+\varphi_{2}(t)-\varphi_{4}(t)+D_{5}(t) \\
& \dot{X}_{B A}(t)=\varphi_{3}(t)-\varphi_{5}(t)+D_{6}(t) \\
& \dot{S}_{I}(t)=D_{7}(t) \\
& \dot{X}_{I}(t)=f_{p}\left[\rho_{4}(t)+\rho_{5}(t)\right]+D_{8}(t) \\
& \dot{S}_{N D}(t)=-\rho_{6}(t)+\rho_{8}(t)+D_{9}(t) \\
& \dot{X}_{N D}(t)=\left(i_{x b}-f_{p} i_{x p}\right)\left[\rho_{4}(t)+\rho_{5}(t)\right]-\rho_{8}(t)+D_{10}(t)
\end{aligned}
$$

where $Y_{a}, Y_{h}, f_{p}, i_{x b}$ and $i_{x p}$ are constant coefficients and $\varphi_{i}(t), i=1, \cdots, 8$ are given by:

$$
\begin{aligned}
\varphi_{1}(t) & =\mu_{h} \frac{X_{D C O}(t)}{K_{d c o}+X_{D C O}(t)} \frac{S_{O}(t)}{K_{o h}+S_{O}(t)} X_{B H}(t) \\
\varphi_{2}(t) & =\mu_{h} \eta_{N O g} \frac{X_{D C O}(t)}{K_{d c o}+X_{D C O}(t)} \frac{S_{N O}(t)}{K_{n o}+S_{N O}(t)} \frac{K_{o h} X_{B H}(t)}{K_{o h}+S_{O}(t)} \\
\varphi_{3}(t) & =\mu_{a} \frac{S_{N H}(t)}{K_{n h, a}+S_{N H}(t)} \frac{S_{O}(t)}{K_{o, a}+S_{O}(t)} X_{B A}(t) \\
\varphi_{4}(t) & =b_{h} X_{B H}(t) \\
\varphi_{5}(t) & =b_{a} X_{B A}(t) \\
\varphi_{6}(t) & =k_{a} S_{N D}(t) X_{B H}(t) \\
\varphi_{7}(t) & =k_{h} X_{D C O}(t) \eta(t) X_{B H}(t) \\
\varphi_{8}(t) & =k_{h} X_{N D}(t) \eta(t) X_{B H}(t) \\
\eta(t) & =\frac{1}{K_{d c o}+\frac{X_{D C O}(t)}{X_{B H}(t)}\left[\frac{S_{O}(t)}{K_{o h}+S_{O}(t)}+\frac{\eta_{h} K_{o h}}{K_{o h}+S_{O}(t)} \frac{S_{N O}(t)}{K_{n o}+S_{N O}(t)}\right]}
\end{aligned}
$$

The input/output balance is defined by:

$$
\begin{aligned}
& D_{1}(t)=D_{\text {in }}(t)\left[X_{D C O, \text { in }}(t)-X_{D C O}(t)\right] \\
& D_{2}(t)=D_{\text {in }}(t)\left[-S_{O}(t)\right]+K q_{a}(t)\left[S_{O, \text { sat }}-S_{O}(t)\right] \\
& D_{3}(t)=D_{\text {in }}(t)\left[S_{N H, \text { in }}(t)-S_{N H}(t)\right] \\
& D_{4}(t)=D_{\text {in }}(t)\left[-S_{N O}(t)\right] \\
& D_{5}(t)=D_{\text {in }}(t)\left[X_{B H, \text { in }}(t)-X_{B H}(t)+\frac{f_{r}\left(1-f_{w}\right)}{f_{r}+f_{w}} X_{B H}(t)\right] \\
& D_{6}(t)=D_{\text {in }}(t)\left[-X_{B A}(t)+\frac{f_{r}\left(1-f_{w}\right)}{f_{r}+f_{w}} X_{B A}(t)\right] \\
& D_{7}(t)=D_{\text {in }}(t)\left[S_{I, \text { in }}(t)-S_{I}(t)\right] \\
& D_{8}(t)=D_{\text {in }}(t)\left[X_{I, \text { in }}(t)-X_{I}(t)+\frac{f_{r}\left(1-f_{w}\right)}{f_{r}+f_{w}}\right] X_{I}(t) \\
& D_{9}(t)=D_{\text {in }}(t)\left[S_{N D, \text { in }}(t)-S_{N D}(t)\right] \\
& D_{10}(t)=D_{\text {in }}(t)\left[X_{N D, \text { in }}(t)-X_{N D}(t)+\frac{f_{r}\left(1-f_{w}\right)}{f_{r}+f_{w}}\right] X_{N D}(t)
\end{aligned}
$$

where $D_{\text {in }}(t)=\frac{q_{\text {in }}(t)}{V}$. The following heterotrophic growth and decay kinetic parameters are used Olsson and Newell [1999]: $\mu_{h}=3.733[1 / 24 h], \mu_{a}=0.3[1 / 24 h], K_{s}=20\left[\mathrm{~g} / \mathrm{m}^{3}\right], f_{s s}=$ $0.79, K_{o h}=0.2\left[\mathrm{~g} / \mathrm{m}^{3}\right], K_{o, a}=0.4\left[\mathrm{~g} / \mathrm{m}^{3}\right], K_{n o}=0.5\left[\mathrm{~g} / \mathrm{m}^{3}\right]$, $K_{n h, a}=1\left[\mathrm{~g} / \mathrm{m}^{3}\right], b_{h}=0.3[1 / 24 h], b_{a}=0.05[1 / 24 h], \eta_{N O g}=$ 0.8 . The stoichiometric parameters are $Y_{h}=0.6\left[\mathrm{~g}\right.$ cell], $Y_{a}=$ $0.24\left[\mathrm{~g}\right.$ cell] $, i_{x b}=0.086[\mathrm{~g} \mathrm{~N}], i_{x p}=0.06[\mathrm{~g} \mathrm{~N}], f_{p}=0.1$ and the oxygen saturation concentration is $S_{O, s a t}=10\left[\mathrm{~g} / \mathrm{m}^{3}\right]$. The fractions $f_{r}$ and $f_{w}: f_{r}=1.1, f_{w}=0.04$ and the tank volume is $V=1333\left[\mathrm{~m}^{3}\right]$.

\subsection{Multi-model description for ASMI}

For lack of space, only the essential points are given in the following. For more details the reader is referred to Nagy et al. [2010]. The idea is to equivalently rewrite the ASM1 model (28) under the MM form (2), i.e. to find $r$, the matrices $A_{i}, B_{i}$, $E_{i}, \Delta A_{i}$ and the weighting functions $\mu_{i}(x, u)$. First, the decision variables are defined as nonlinearities of the system (28):

$$
\begin{aligned}
z_{1}(x, u) & =\frac{q_{\text {in }}(t)}{V} \\
z_{2}(x, u) & =\frac{X_{D C O}(t)}{K_{d c o}+X_{D C O}(t)} \frac{S_{O}(t)}{K_{o h}+S_{O}(t)} \\
z_{3}(x, u) & =\frac{1}{K_{o, a}+S_{O}(t)} \frac{S_{N H}(t)}{K_{n h, a}+S_{N H}(t)} X_{B A}(t) \\
z_{4}(x, u) & =S_{N H}(t) \\
z_{5}(x, u) & =\frac{X_{D C O}(t)}{K_{d c o}+X_{D C O}(t)} \frac{S_{N O}(t)}{K_{n o}+S_{N O}(t)} \frac{K_{o h}}{K_{o h}+S_{O}(t)} \\
z_{6}(x, u) & =X_{D C O}(t) \eta(t)
\end{aligned}
$$

Remark 4.1. In order to avoid potential infeasible LMI solutions for the observer design, the number of decision variables should be reduced. Small dynamic variations and values can 
be observed for $z_{3}, z_{5}$ and $z_{6}$ compared to the other decision variables, which allows to consider their means $\tilde{z_{3}}, \tilde{z_{5}}$ and $\tilde{z_{6}}$ for the construction of the MM form (2).

A convex polytopic transformation is performed for all the decision variables $(j=1,2,4)$, as follows:

$$
z_{j}(x, u)=F_{j, 1}\left(z_{j}(x, u)\right) z_{j, 1}+F_{j, 2}\left(z_{j}(x, u)\right) z_{j, 2}
$$

where the scalars $z_{j, 1}, z_{j, 2}$ are respectively the maxima and minima of $z_{j}(x, u)$ and $F_{j, 1}\left(z_{j}\right), F_{j, 2}\left(z_{j}\right)$ are defined by

$$
F_{j, 1}\left(z_{j}\right)=\frac{z_{j}(x, u)-z_{j, 2}}{z_{j, 1}-z_{j, 2}}, F_{j, 2}\left(z_{j}\right)=\frac{z_{j, 1}-z_{j}(x, u)}{z_{j, 1}-z_{j, 2}}
$$

By multiplying the functions $F_{j, \sigma_{i}^{j}}\left(z_{j}(x, u)\right)$, the $r=8$ weighting functions are obtained:

$$
\mu_{i}(z)=F_{1, \sigma_{i}^{1}}\left(z_{1}(u)\right) F_{2, \sigma_{i}^{2}}\left(z_{2}(x, u)\right) F_{4, \sigma_{i}^{4}}\left(z_{4}(x, u)\right)
$$

The constant matrices $A_{i}, B_{i}$ and $E_{i}$ defining the 8 sub-models are given by:

$$
\begin{aligned}
& A_{i}=A\left(z_{1, \sigma_{i}^{1}}, z_{2, \sigma_{i}^{2}}, z_{4, \sigma_{i}^{4}}\right) \\
& B_{i}=B\left(z_{1, \sigma_{i}^{1}}\right) \\
& E_{i}=E\left(z_{1, \sigma_{i}^{1}}\right), i=1, \ldots, 8, j=1,2,4
\end{aligned}
$$

where the matrices $A\left[a_{i, j}\right] \in \mathbb{R}^{10 \times 10}, B\left[b_{i, j}\right] \in \mathbb{R}^{10 \times 6}$ and $E\left[e_{i, j}\right] \in \mathbb{R}^{10 \times 1}$ are defined by the following compounds: $a_{1,1}(x, u)=a_{3,3}(x, u)=a_{4,4}(x, u)=a_{7,7}(x, u)=a_{9,9}(x, u)$ $=b_{1,1}(x, u)=b_{7,3}(x, u)=b_{8,4}(x, u)=b_{9,5}(x, u)=b_{10,6}(x, u)=$ $e_{3,1}(x, u)=-z_{1}(u), b_{2,2}=K S_{O, s a t}$ and

$$
\begin{aligned}
a_{1,5}(x, u) & =-\frac{\mu_{h}}{Y_{h}} z_{2}(x, u)+\left(1-f_{p}\right) b_{h}-\frac{\mu_{h} \eta_{N O g}}{Y_{h}} \tilde{z_{5}} \\
a_{1,6}(x, u) & =\left(1-f_{p}\right) b_{a} \\
a_{2,2}(x, u) & =-z_{1}(u)-K q_{a}-\frac{4.57-Y_{a}}{Y_{a}} \mu_{a} \tilde{z_{3}} \\
a_{2,5}(x, u) & =\frac{\left(Y_{h}-1\right) \mu_{h}}{Y_{h}} z_{2}(x, u) \\
a_{3,2}(x, u) & =-\left(i_{x b}+\frac{1}{Y_{a}}\right) \mu_{a} \tilde{z_{3}} \\
a_{3,5}(x, u) & =\left(i_{x b}-f_{p} i_{x p}\right) b_{h}-i_{x b} \mu_{h}\left[z_{2}(x, u)+\eta_{N O g} \tilde{z_{5}}\right] \\
a_{3,6}(x, u) & =\left(i_{x b}-f_{p} i_{x p}\right) b_{a} \\
a_{4,2}(x, u) & =\frac{1}{Y_{a}} \mu_{a} \tilde{z} \\
a_{4,5}(x, u) & =\frac{Y_{h}-1}{2.86 Y_{h}} \mu_{h} \eta_{N O g} \tilde{z_{5}} \\
a_{5,5}(x, u) & =\mu_{h} z_{2}(x, u)-b_{h}+\mu_{h} \eta_{N O g} \tilde{z_{5}}+z_{1}(u) \tilde{f} \\
a_{6,2}(x, u) & =\mu_{a} \tilde{z_{3}} \\
a_{6,6}(x, u) & =z_{1}(u) \tilde{f}-b_{a} \\
a_{8,5}(x, u) & =f_{p} b_{h} \\
a_{8,6}(x, u) & =f_{p} b_{a} \\
a_{8,8}(x, u) & =\tilde{f} z_{1}(u) \\
a_{9,5}(x, u) & =-k_{a} z_{4}(x, u)+k_{h} \tilde{z_{6}} \\
a_{10,5}(x, u) & =\left(i_{x b}-f_{p} i_{x p}\right) b_{h}-k_{h} \tilde{z_{6}} \\
a_{10,6}(x, u) & =\left(i_{x b}-f_{p} i_{x p}\right) b_{a} \\
a_{10,10}(x, u) & =\tilde{f} z_{1}(u) \\
&
\end{aligned}
$$

where $\tilde{f}=\left[\frac{f_{r}\left(1-f_{w}\right)}{f_{r}+f_{w}}-1\right]$. The rest of matrices compounds not mentioned here are zero. Using this, the reduced MM form (Remark 4.1) of the ten state reduced ASM1 is completed. It should be said that the reduced MM accurately represents the ASM1 (28), excepting the two concentrations $S_{N O}$ and $X_{N D}$ for which a quite good representation is nevertheless obtained, as seen in figure 1 .
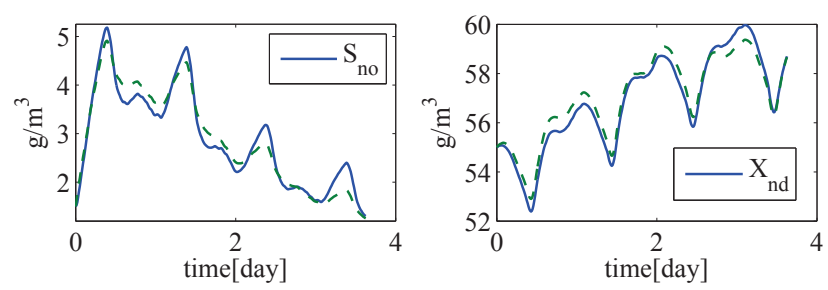

Fig. 1. Comparison between the reduced $\mathrm{MM}$ (dashed) and ASM1(solid)

In order to take into account parameter uncertainties on $b_{H}$ and $b_{A}$, the MM structure is slightly modified. These parameters appear in the coefficients $a_{15}, a_{16}, a_{35}, a_{36}, a_{55}$ and $a_{66}$ in (35), allowing to separate the uncertain part $\Delta A(t)$ from the known one $A(t)$. The parameter variation on $b_{H}$ (resp. $b_{A}$ ) is of $20 \%$ (resp. $25 \%$ ) of its nominal value, i.e. $b_{H} \in$ $[0.25 ; 0.35]$ (resp. $\left.b_{A} \in[0.04 ; 0.06]\right)$. The uncertain terms of ASM1 $\Delta B_{i}(t)=0$ and $\Delta A_{i}(t)=\Delta A(t)$ are written under the form $\Delta A(t)=M^{a} F_{a}(t) N^{a}$ where:

$$
\begin{aligned}
M^{a} & =\left[\begin{array}{llllllllll}
1 & 0 & 1 & 0 & 1 & 0 & 0 & 1 & 0 & 1 \\
1 & 0 & 1 & 0 & 0 & 1 & 0 & 1 & 0 & 1
\end{array}\right]^{T} \\
F_{a}(t) & =\left[\begin{array}{ccccc}
\Delta b_{H}(t) & 0 & 0 & \\
0 & & \Delta b_{A}(t)
\end{array}\right] \\
N^{a} & =\left[\begin{array}{llllllllll}
0 & 0 & 0 & 0 & 1 & 0 & 0 & 0 & 0 & 0 \\
0 & 0 & 0 & 0 & 0 & 1 & 0 & 0 & 0 & 0
\end{array}\right]
\end{aligned}
$$

The data used for simulation are generated with the complete ASM1 model with 13 state variables Henze et al. [1987], in order to represent a realistic behavior of a WWTP. Applying the Theorem 1 for $q=4$ the observer (5) is designed by finding positive scalars $\varepsilon_{1 i}, \varepsilon_{2 i}$, positive definite matrices $P_{1}$ and $P_{2}$ and matrices $\bar{P}_{i}(i=1, \cdots, 8)$-that are not given here due to space limitation- such that the convergence conditions, given in Theorem 1 hold. The value of the attenuation rate from the known and unknown inputs $u(t)$ and $d(t)$ to the state and fault estimation error $e_{a}(t)$ is $\bar{\gamma}=0.52$. The positive scalars gathered in vectors are $\varepsilon_{1}=[0.3313,0.3339,0.3428,0.3424,0.3064$, $0.3099,0.3167,0.3185], \varepsilon_{1}=[0.2767,0.2766,0.2772,0.2777$, $0.2772,0.2763,0.2778,0.2788]$. A comparison between the actual state variables, the unknown inputs and their respective estimates is depicted in figures 2 and 3. In fig. 2, the estimation errors for $S_{N O}$ and $X_{N D}$ are in part generated by the reduction made on the MM (see the Remark 4.1).

\section{CONCLUSIONS AND FUTURE WORKS}

\subsection{Conclusions}

The MM approach provides the state of the art solutions to many problems involving estimation, filtering, control, and/or modeling. As a major advantage of the MM against a general nonlinear model there is the possibility to use many tools developed in the framework of linear system. The paper propose the observer synthesis for uncertain nonlinear systems affected by unknown inputs described by the multi-model formulation with 

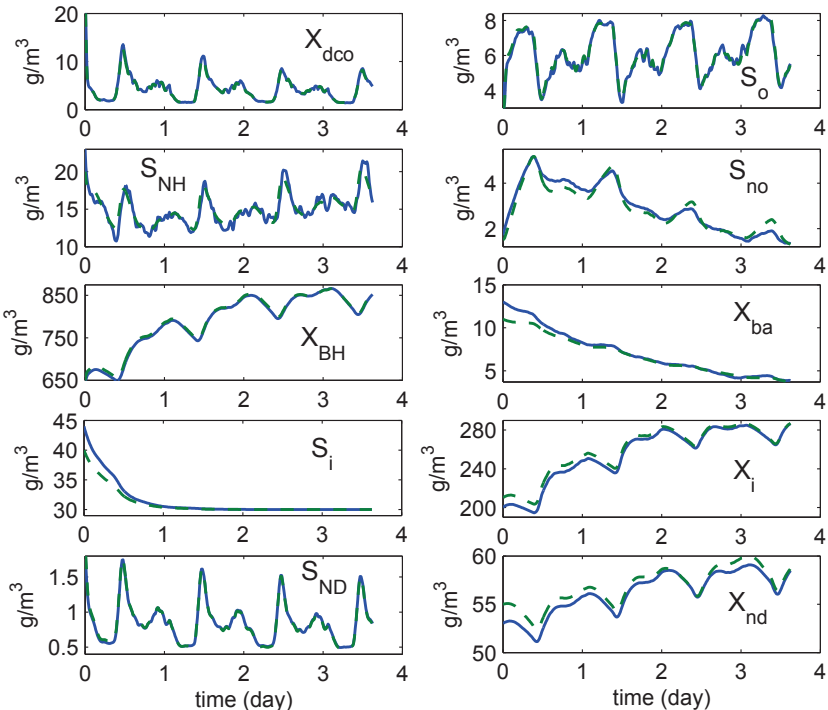

Fig. 2. State estimation using PMI observer (dashed) for ASM1 (solid)

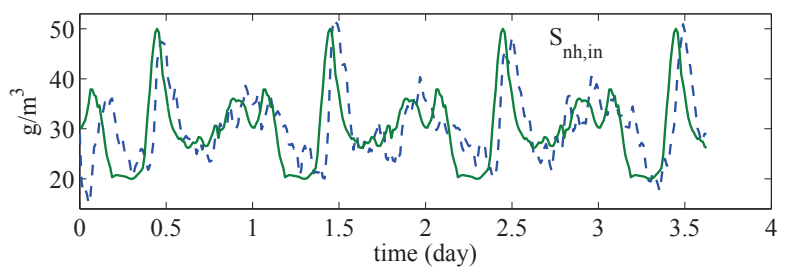

Fig. 3. Unknown input estimation using PMI observer (dashed) for complete ASM1 (solid)

unmeasurable decision variables. The application to a wastewater treatment plant model, which is an uncertain nonlinear system affected by unknown inputs is afterwards realized in order to prove the performance of the proposed observer.

\subsection{Future Works}

The future developments are numerous and among them one can think of the choice of adapted structures of the local models with specific properties, such as diagnosability. Another important problem concerns the use of MM and PMI observers to detect and isolate sensor and actuator faults in the system.

\section{REFERENCES}

G. Z. Angelis. System Analysis, Modeling and Control with Polytopic Linear Models. Technische Universiteit Eindhoven, 2001.

P. Bergsten, R. Palm, and D. Driankov. Observers for TakagiSugeno fuzzy systems. IEEE Transactions on Systems, Man and Cybernetics, 32:114-121, 2002.

S. Boyd, L. El Ghaoui, E. Feron, and V. Balakrishnan. Linear matrix inequalities in system and control theory. Studies in Applied and Numerical Mathematics, Philadelphia, 1994.

W. Chen and M Saif. Design of a TS based fuzzy nonlinear unknown input observer with fault diagnosis applications. In American Control Conference, New York, USA, 2007.
J.B. Coop, editor. Description and Simulator Manual, chapter The COST Simulation Benchmark. Office for Official of the European Communities, Luxembourg, 2002.

Z. Gao and D. Ho. Proportional multi-integral observer for descriptor systems with measurement output disturbances. IEEE Proceeding - Control theory and application, 151(3): 279-288, 2004.

M. Henze, C. P. Leslie Grady Jr, W. Gujer, G. v. R. Marais, and T. Matsuo. Activated Sludge Model no.1. I.A.W.Q. Scientific and Technical Report No.1. Technical report, I.A.W.Q., London, UK, 1987.

D. Ichalal, B. Marx, J. Ragot, and D. Maquin. Simultaneous state and unknown inputs estimation with PI and PMI observers for Takagi-Sugeno model with unmeasurable premise variables. In Mediterranean Conference on Control and Automation, Thessaloniki, Greece, 2009.

D. Ichalal, B. Marx, J. Ragot, and D. Maquin. Fault tolerant control for takagi-sugeno systems with unmeasurable premise variables by trajectory tracking. In IEEE International Symposium on Industrial Electronics, Bari, Italy, 2010.

G. Jiang, S. Wang, and W. Song. Design of observer with integrators for linear systems with unknown input disturbances. Electronics Letters, 36(13):1168-1169, 2000.

T. Johansen, R. Shorten, and R. Murray-Smith. On the interpretation and identification of dynamic Takagi-Sugeno fuzzy models. IEEE Transactions on Fuzzy Systems, 8(3):297-313, 2000 .

D. Koenig. Unknown input PMI observer design for linear descriptor systems: application to state and fault estimation. IEEE Transactions on Automatic Control, 50(2):212-217, 2005.

D. Koenig and S. Mammar. Design of proportional-integral observer for unknown input descriptor systems. IEEE Transactions on Automatic Control, 47(12):2057-2062, 2002.

B. Marx, D. Koenig, and J. Ragot. Design of observers for Takagi-Sugeno descriptor systems with unknown inputs and application to fault diagnosis. IET Control Theory and Applications, 1(5):1487-1495, 2007.

R. Murray-Smith and T. A. Johansen. Multiple model approaches to modeling and control. Taylor \& Francis, London, 1997.

A. M. Nagy, G. Mourot, B. Marx, G. Schutz, and J. Ragot. Systematic multi-modeling methodology applied to an activated sludge reactor model. Industrial \& Engineering Chemistry Research, 49(6):2790-2799, February, 162010.

A.M. Nagy-Kiss, B. Marx, G. Mourot, G. Schutz, and J. Ragot. Observers design for uncertain Takagi-Sugeno systems with unmeasurable premise variables and unknown inputs. Application to a wastewater treatment plant. Journal of Process Control, 21(7):1105-1114, 2011. ISSN 0959-1524.

G. Olsson and B. Newell. Wastewater Treatment Systems. Modelling, Diagnosis and Control. IWA Publishing, 1999.

I. Y. Smets, J. V. Haegebaert, R. Carrette, and J. F. Van Impe. Linearization of the activated sludge model ASM1 for fast and reliable predictions. Water Research, 37:1831-1851, 2003.

K. Tanaka and H. O. Wang. Fuzzy Control System Design and analysis. A Linear Matrix Inequality Approach. John Wiley \& Sons Inc., 2001. 